\title{
A pós-graduação em Medicina Veterinária no Estado de São Paulo: Faculdade de Medicina Veterinária da Universidade de São Paulo - 1965
} a 1970*

\begin{abstract}
Post-graduation in Veterinary Medicine in the State of São Paulo, Brazil: School of Veterinary Medicine of the University of São Paulo - 1965 to 1970
\end{abstract}

\section{El posgrado en Medicina Veterinaria en el Estado de São Paulo, Brasil: Facultad de Medicina Veterinaria de la Universidad de São Paulo - 1965 a 1970}

\section{Eduardo Harry Birgel ${ }^{1}$}

\section{Resumo}

Após abordar a comemoração, em 2004, dos "70 Anos de Criação e Implantação da Universidade de São Paulo" e as festividades, paralelas que a Pró-Reitoria de Pós-Graduação promoveu sobre os "35 Anos da Pós-Graduação na USP", argumenta que as comemorações da Pós-Graduação foram promovidas e divulgadas com registro errado de data. Destaca que os primeiros títulos de Mestre em Medicina Veterinária foram obtidos na Faculdade de Medicina Veterinária da Universidade de São Paulo, São Paulo, SP, no período de 1965 a 1970. Apresenta anexo ao artigo a relação dos médicos veterinários que obtiveram o título de Mestre nesse período.

Palavras-chave: Universidades, história. Escolas de veterinária, história. Educação de pós-graduação, história. Médicos veterinários. Diretório.

'Professor Titular Aposentado da Faculdade de Medicina Veterinária e Zootecnia da Universidade de São Paulo. Pesquisador Científico Nível IA do Conselho Nacional de Desenvolvimento Científico e Tecnológico (CNPq). CRMV-SP 00018

* Reminiscência histórica do ensino de pós-graduação em Medicina Veterinária, por solicitação do CRMV-SP. Anexo ao artigo a relação dos médicos veterinários que obtiveram o título de Mestre no periodo de 1965 a 1970 


\section{Relação de médicos veterinários que obtiveram Título de Mestre pela Faculdade de Medicina Veterinária da Universidade de São Paulo (FMV/USP), no período de 1965 a 1970}

\begin{abstract}
Os nomes dos médicos veterinários que constam dessa lista, obtiveram o título acadêmico de mestre após concluírem com aproveitamento os cursos de pós-graduação em Reprodução Animal (1965) e Cirurgia Bovina (1967) e, após apresentação de dissertação de mestrado, foram pioneiros e fizeram jus ao título de Mestre em Medicina Veterinária. A relação foi feita, baseada nas informações da publicação: SISTEMA INTEGRADO DE BIBLIOTECAS. Catálogo de teses: 1934-1984. 2. ed. Série III. Ciências biológicas. Parte 2. São Paulo: SIBI, 1987.
\end{abstract}

Nesse momento, cabe serem feitos alguns comentários a respeito da importância desses pioneiros cursos de mestrado para a Ciência Veterinária e o significado desses mestres para o ensino da Medicina Veterinária no Estado de São Paulo.

Caso fossem utilizados os contestáveis critérios da Coordenação de Aperfeiçoamento de Pessoal de Nível Superior (CAPES) para avaliação dos cursos de pós-graduação stricto sensu brasileiros, seguramente o conceito seria um dos melhores, pois, considerando os docentes da Universidade de São Paulo, pode-se afirmar:

- Os títulos foram obtidos com menos de 24 meses de atividade (a CAPES valoriza mais o tempo de duração dos Cursos do que o valor científico da dissertação ou tese);

- Os 27 pós-graduandos que se tornaram docentes da USP após dois anos apresentaram tese de doutoramento (a produtividade científica era intensa e de qualidade);

- Desses 27 docentes da USP, 13 completaram a carreira docente nessa instituição $(77 \%)$, tornadose professores titulares (grande capacidade formadora de novos pesquisadores);
- Esses 13 docentes ocuparam, na Faculdade de Medicina Veterinária e Zootecnia (FMVZ/USP), importantes cargos administrativos - oito Chefias de Departamentos, três Diretorias do Centro Interdepartamental de Zootecnia e Indústria Pecuária da FMVZ/USP (CIZIP) e duas Diretorias da FMVZ/USP (desprendimento pessoal dedicando-se a atividades administrativas);

- Esses docentes foram os implantadores da atual pós-graduação da FMVZ/USP (iniciativa e aplicação no desenvolvimento de novos projetos).

No caso dos mestres desse curso, que eram ou se tornaram docentes da Faculdade de Medicina Veterinária e Zootecnia da Universidade Estadual Paulista (FMVZ/UNESP), Campus de Botucatu, SP, verifica-se o seguinte quadro:

- Sete dos 37 pós-graduandos (19\%) pertenceram ao quadro funcional da UNESP;

- Seis foram professores titulares (86\%);

- Quatro ocuparam, seguramente, a chefia de departamentos (67\%);

- Dois foram diretores da FMVZ/UNESP, Campus de Botucatu, SP (33\%).

Seguramente a atividade e dedicação destes docentes foi fator significativo para a implantação, evolução e sucesso dessa tradicional e reconhecida instituição de ensino de graduação e pós-graduação da Medicina Veterinária.

Na relação exposta a seguir (Quadro 1), além de constar o nome do médico veterinário mestre, incluiu-se o ano de aprovação da dissertação de mestrado, o local em que exercia a profissão e a função final de sua vida profissional, informando ainda se o colega é aposentado ou falecido. 
BIRGEL, E. H. A pós-graduação em Medicina Veterinária: FMVZ/USP - 1965 a 1970. / Post-graduation in Veterinary Medicine: FMVZ USP - 1965 to 1970. / El posgrado en Medicina Veterinaria: FMVZ/USP - 1965 a 1970. Rev. Educ. Contin. CRMV-SP / Contin. Educ.

J. $\boldsymbol{C R M V - S P , ~ S a ̃ o ~ P a u l o , ~ v . ~ 7 , ~ n . ~ 1 / 3 , ~ p . ~ 7 9 - 8 2 , ~} 2004$.

Quadro 1 - Relação dos médicos veterinários que obtiveram o título de mestre entre 1966 e 1970

\begin{tabular}{|c|c|c|c|c|c|c|}
\hline \multirow[t]{2}{*}{ Nome } & \multirow[t]{2}{*}{ Ano/Titulo } & \multirow[t]{2}{*}{ Instituição de exercicio profissional } & \multicolumn{4}{|c|}{ Estado atual* } \\
\hline & & & $\mathrm{F}$ & A & $\mathrm{AC}$ & PA \\
\hline Sylvio Ferri & 1966 & Instituto de Ciências Biomédicas/USP - Professor Titular & & A & & \\
\hline Antonio Alberto D'Errico & 1967 & FMVZ/USP - Professor Adjunto & & A & & \\
\hline Antonio Fernandes Filho & 1967 & $\begin{array}{l}\text { FMVZ/USP - Professor Titular. Coordenador do Curso de } \\
\text { Veterinária da Universidade de Santo Amaro }\end{array}$ & & A & & \\
\hline Arli de Campos Pacheco & 1967 & FMVZ/UNESP - Botucatu. Professor Titular & & A & & \\
\hline Arlindo Garcia Moreno & 1967 & FMVZ/USP - Professor Adjunto. Diretor do CIZIP & & A & & \\
\hline Bário Raymundo Cirne & 1967 & Instituto de Ciências Biomédicas/USP. & & A & & \\
\hline Bruno Soerensen Cardoso & 1967 & FMVZ/UNESP - Botucatu. Universidade de Marília & & & & \\
\hline Eduardo Harry Birgel & 1967 & $\begin{array}{l}\text { FMVZ/USP - Professor Titular e ex-Chefe do Departamento } \\
\text { de Clínica Médica. Responsável pela implantação da pós-graduação } \\
\text { no Departamento }\end{array}$ & & & $\mathrm{AC}$ & \\
\hline Esleibe Ghion & 1967 & FMVZ/USP - Professor Titular. Ex-Diretor do CIZIP & & A & & \\
\hline Flávio Prada & 1967 & $\begin{array}{l}\text { FMVZ/USP - Professor Titular. Ex-Chefe do Departamento } \\
\text { de Clinica Médica. Interventor Judicial do CRMV-SP }\end{array}$ & & A & & \\
\hline Gilberti Moreno & 1967 & FMVZ/USP. FMVZ/UNESP - Botucatu. Professor Titular & & A & & \\
\hline Homero Moraes Barros & 1967 & FMVZ/USP. FMVZ/UNESP - Botucatu. Professor Titular & & A & & \\
\hline João Silva Marcondes Veiga & 1967 & FMVZ/USP - Docente & $\mathrm{F}$ & & & \\
\hline José Cezar Panetta & 1967 & $\begin{array}{l}\text { FMVZ/USP - Professor Titular e ex-Diretor. } \\
\text { Ex-presidente do CRMV-SP }\end{array}$ & & A & & \\
\hline Karin Gürsching & 1967 & FMVZ/USP - Ambulatório. & & & & PA \\
\hline Leonardo Miranda de Araújo & 1967 & FMVZ/USP - Professor Titular. Vice-Diretor do CIZIP & $\mathrm{F}$ & & & \\
\hline $\begin{array}{l}\text { Manuel Alberto da Silva Castro } \\
\text { Portugal }\end{array}$ & 1967 & Instituto Biológico & & A & & \\
\hline Marina Therezinha & & & & & & \\
\hline Refatti de Moura & 1967 & Instituto de Ciências Biomédicas/USP. FMVZ/USP. & & & & PA \\
\hline Mario Annunziata & 1967 & FMVZ/USP & $\mathrm{F}$ & & & \\
\hline Mário Nakano & 1967 & Instituto Biológico & & A & & \\
\hline Mikiko Tokumaru & 1967 & Instituto de Ciências Biomédicas/USP - Docente & & A & & \\
\hline Oziel Bizzuti & 1967 & Instituto de Ciências Biomédicas/USP & $\mathrm{F}$ & & & \\
\hline Renato Campanarut Barnabé & 1967 & FMVZ/USP - Professor Titular. Diretor do CIZIP & & & $\mathrm{AC}$ & \\
\hline Roberto Grecchi & 1967 & FMVZ/USP - Professor Titular & & A & & \\
\hline Rubens Gonçalves Amaro & 1967 & Instituto de Ciências Biomédicas/USP. Instituto Biológico & F & & & \\
\hline Sérgio de Moraes & 1967 & FMVZ/USP. Instituto de Ciências Biomédicas/USP - Professor Titular & & A & & \\
\hline Valêncio José de Mattos Campos & 1967 & FMVZ/UNESP - Botucatu. Professor Titular & & A & & \\
\hline Vicente Borelli & 1967 & $\begin{array}{l}\text { FMVZ/USP - Professor Titular e ex-Diretor. Coordenador } \\
\text { do Curso de Veterinária da Universidade Paulista }\end{array}$ & & A & & \\
\hline Victor Elias Cury José Kerbauy & 1967 & FMVZ/USP - Docente. & & & & PA \\
\hline Walter Nazário & 1967 & Instituto Biológico & $\mathrm{F}$ & & & \\
\hline Cid Figueiredo & 1969 & FMVZ/UNESP - Botucatu. Professor Titular & & A & & \\
\hline Paulo Alves de Siqueira & 1969 & Instituto Biológico & & A & & \\
\hline Antonio Matera & 1970 & FMVZ/USP - Professor Titular & & A & & \\
\hline José Peduti Neto & 1970 & FMVZ/USP - Professor Adjunto & $\mathrm{F}$ & & & \\
\hline Raul Gastão Muciolo & 1970 & FMVZ/USP - Professor Adjunto & & A & & \\
\hline Waldir Gandofi & 1970 & FMVZ/UNESP - Botucatu. Professor Titular e ex-Diretor & & A & & \\
\hline Walter Spicciati & 1970 & FMVZ/USP - Docente & $\mathrm{F}$ & & & \\
\hline
\end{tabular}

* As informações sobre o status dos médicos veterinários não estão atualizadas, e nem são oficiais, pois alguns já faleceram (recentemente) e outros exercem atividades docentes em Instituições de Ensino Superior com vínculo administrativo privado. O CRMV-SP gostaria de receber informações complementares para registro histórico.

**A $=$ Aposentado; $\quad \mathrm{AC}=$ Aposentado pela compulsória; $\mathrm{F}=$ Falecido; $\mathrm{PA}=$ Profissional autônomo 


\section{Abstract}

Having addressed the celebration, in 2004, of the "70 Years of the Creation and Implantation of the University of São Paulo", and the other festivities promoted by the Post-graduation Vice-presidency on account of the "35 Years of Post-Graduation at USP", it argues that the Post-Graduation celebrations were promoted and disclosed with wrong date registers. It points out that the first Master titles in Veterinary Medicine were obtained at the School of Veterinary Medicine of the University of São Paulo, São Paulo, SP, Brazil, between 1965 and 1970. The veterinarians who obtained the Master title during this period are listed in an annex to this document.

Keywords: Universities, history. Schools, veterinary, history. Education, graduate, history.

Veterinarians. Directory.

\section{Resumen}

Después de abordar la conmemoración en 2004 de los "70 Años de Creación e Implantación de la Universidad de São Paulo" y las festividades paralelas que el Rectorado de Posgrado realizó sobre los "35 Años del Posgrado en la USP", argumenta que las conmemoraciones del Posgrado fueron promovidas y divulgadas con un registro equivocado de fecha. Destaca que los primeros títulos del Máster en Medicina Veterinaria se obtuvieron en la Facultad de Medicina Veterinaria de la Universidad de São Paulo, São Paulo, SP, Brasil, en el periodo de 1965 a 1970. Presenta como adjunto al artículo, la relación de los médicos veterinarios que obtuvieron el título en el periodo.

Palabras-clave: Universidades, historia. Escuelas de veterinaria, historia. Educación de posgrado, historia. Veterinarios. Directorio.

Endereço / Addresss / Dirección: 